\title{
Comunidades infaunales asociadas a la almeja Ensis macha: impacto de la pesca por hidrojet
}

\author{
María M. MendeZ; Jorgelina Robledo \& Silvina Van der Molen²凶 \\ ${ }^{1}$ Laboratorio de Reproducción y Biología Integrativa de Invertebrados Marinos (IBIOMAR-CONICET). Puerto Madryn, \\ Chubut, Argentina. ${ }^{2}$ Instituto de Biología de Organismos Marinos (CONICET). Puerto Madryn, Chubut, Argentina.
}

\begin{abstract}
Resumen. La almeja navaja (género Ensis) se explota en todo el mundo mediante una variedad de artes de pesca. Los impactos ambientales asociados a estas pesquerías incluyen cambios en las características físicas del sedimento y en la columna de agua, también efectos sobre las comunidades asociadas y sobre las mismas especies objetivo. El impacto producido depende principalmente del arte de pesca, del tipo de hábitat y de la intensidad de pesca. Los marisqueros del Golfo San José (Península Valdés, Chubut) emplean el hidrojet, una técnica que implica la inyección de agua en el sustrato para extraer navajas en distintos bancos. Si bien la explotación de este recurso en el Golfo San José aún es incipiente, resulta clave el estudio del impacto de esta pesquería para poder establecer medidas de manejo que se adecuen a la técnica empleada y al ambiente del Golfo, antes de que la pesquería se desarrolle a mayor escala. Así, el objetivo de este estudio fue evaluar de forma experimental el impacto de la pesca de la almeja navaja Ensis macha mediante el uso de la técnica hidrojet sobre las comunidades bentónicas asociadas a los bancos de esta almeja en el Golfo San José. Para ello, se comparó la composición de la fauna bentónica en dos bancos de navajas, uno con actividad extractiva y otro control, en el momento de la pesca y al año de realizada la actividad. Las comparaciones mostraron que en el tiempo inicial no se observaron diferencias entre el banco impactado y el control, mientras que luego de un año de realizada la actividad de pesca, las diferencias en la composición de las comunidades de ambos bancos fueron significativas. Así, el banco control se caracterizó por la presencia de poliquetos, anfípodos y estomatópodos, mientras que los moluscos bivalvos y gasterópodos fueron los grupos más representativos en el banco impactado. Los resultados hallados son útiles para sugerir medidas de conservación y gestión que eviten la degradación de la biodiversidad local.
\end{abstract}

[Palabras clave: Patagonia, suficiencia taxonómica]

\begin{abstract}
Aвstract. Infaunal communities associated to the razor clam Ensis macha: water-jet fishing impact. Razor clams (genus Ensis) are exploited worldwide through a variety of fishing gears. The environmental impacts produced by these fisheries include changes in the sediment physical characteristics and the water column, as well as the effects on the associated communities and on the target species. The impact mainly depends on the fishing gear, the type of habitat and the fishing intensity. San José gulf (Península Valdés, Chubut) fishermen use the water-jet technique, which involves the injection of water into the substrate to extract the razor clams. Although the exploitation of this resource in the San José gulf is still incipient, it is important to study the impact of this fishery in order to establish appropriate management measures to related the technique used and to the gulf environment, before the fishery grows. Thus, the aim of this study was to experimentally evaluate the impact of Ensis macha water-jet fishing on the benthic communities associated with its beds in San José gulf. The composition of the benthic fauna was compared in two beds, one with extractive activity and another as control, at the time of fishing and after a year. Comparisons showed no differences in the initial time between the impacted bed and the control one, while after a year of the fishing activity the differences in the communities' composition of both beds were significant. The control bed was characterized by polychaetes, amphipods and stomatopods, while the bivalves and gastropods were representative of the impacted bed. The results found are useful in suggesting conservation and management measures to avoid degradation of local biodiversity.
\end{abstract}

\section{INTRODUCCIÓN}

El efecto de las pesquerías sobre los ecosistemas marinos y estuariales está bien documentado (Robinson and Richardson 1998; Bishop et al. 2005). Este efecto depende principalmente del arte de pesca utilizado, del tipo de hábitat y de la intensidad de pesca, y afecta tanto a las características físicas del sedimento y de la columna de agua, como a las comunidades asociadas y a la especie objetivo de la pesca. Por lo tanto, resulta clave que, a

\section{Editor asociado: Pedro Daleo}

$\triangle$ svandermolen@cenpat-conicet.gob.ar medida que se desarrollen nuevas técnicas de extracción y nuevas pesquerías, se realicen estudios de impacto que aporten información en las futuras decisiones de manejo (Tuck et al. 2000). Dado que la complejidad estructural del hábitat es crucial en los ambientes bentónicos, la alteración de la misma tendrá efectos no sólo sobre su diversidad biológica, sino también sobre las pesquerías, puesto que muchas especies comerciales se alimentan de 
las especies que componen el bentos (Tuck et al. 2000; revisión de Gaspar et al. 2008). La dirección del efecto sobre las distintas especies del bentos (i.e., si aumenta o disminuye la abundancia de los distintos organismos) suele ser variable y estar relacionada con los hábitos de cada grupo (Tuck et al. 2000). Por ejemplo, Tuck y colaboradores (2000) registraron un aumento inmediato en la abundancia de anfípodos y una disminución en los poliquetos en sitios disturbados por la pesca, y sugieren que esto puede estar asociado a la movilidad diferencial de ambos grupos.

Varias especies de almejas navajas se explotan comercialmente en todo el mundo. Es un recurso relevante en varios países de Europa, tanto en el Atlántico como en el Mediterráneo, en Estados Unidos (costas pacífica y atlántica) y en Chile (Gaspar et al. 2011). En Argentina sólo existen datos sobre la pesca de navajas en el Golfo San Matías (provincia de Río Negro) (Morsán and Ciocco 2011). Aunque no hay actividades extractivas oficialmente reportadas, también se las pesca en el Golfo San José (Provincia de Chubut).

Las almejas navajas del género Ensis son un grupo de bivalvos infaunales que habitan fondos blandos, están altamente especializados y presentan una concha alargada y comprimida que les facilita la penetración en el sedimento. La almeja navaja Ensis macha, única especie en el extremo sur de Sudamérica, se distribuye en la costa del Océano Pacífico (desde la Bahía de

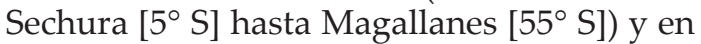
la costa Atlántica (desde el Golfo San Matías [41 ${ }^{\circ}$ S] hasta Tierra del Fuego [54 S]) (Lasta et al. 1998; Espinoza et al. 2010). La almeja navaja es un bivalvo filtrador suspensívoro que habita fondos fango-arenosos y que puede ser encontrada desde el submareal somero hasta profundidades cercanas a los $55 \mathrm{~m}$, formando agregaciones denominadas bancos (Ciocco 1995). Dado que las navajas viven enterradas a cierta profundidad en el sedimento y que presentan un comportamiento de enterramiento en huida que les permite alcanzar profundidades mayores a $30 \mathrm{~cm}$ (Márquez, comunicación personal), los métodos requeridos para una extracción rentable precisan, en algunos casos, de una remoción eficaz del sustrato. En el caso de los bancos de navajas del Golfo San José, se ha implementado la pesca mediante remoción del sustrato utilizando inyección de agua a presión desde las embarcaciones, una técnica conocida como hidrojet (Ciocco 2000).
En las pesquerías de navajas se ha estudiado el impacto de determinadas artes de pesca como dragas mecánicas e hidráulicas (Gaspar et al. 2008). Sin embargo, los pocos trabajos realizados sobre el impacto del hidrojet arrojan resultados que incluyen desde una lenta recuperación de la fauna asociada hasta serios efectos en el reclutamiento de la especie objetivo. Se destaca la necesidad de contar con estudios de impacto locales (Tuck et al. 2000).

El Golfo San José fue declarado Patrimonio de la Humanidad por la UNESCO en el año 1993 debido a su riqueza faunística y paisajística. Sin embargo, a pesar de su importancia ecológica, la fauna bentónica de este Golfo ha sido poco estudiada. En un estudio preliminar, Robledo y Van der Molen (2012) encontraron que las comunidades asociadas a los bancos de navajas del Golfo San José están compuestas por moluscos, crustáceos, poliquetos, foraminíferos y, en menor medida, equinodermos. El propósito de los estudios de impacto de la pesca es evaluar si la actividad extractiva produce cambios en el ambiente, para poder determinar qué componentes son realmente afectados y determinar la magnitud de dichos efectos. En el presente trabajo evaluamos de forma experimental el impacto de la pesca de almejas navajas mediante el uso de la técnica de hidrojet sobre las comunidades bentónicas asociadas a los bancos en el Golfo San José.

\section{Materiales y Métodos}

\section{Sitio de estudio}

El estudio se llevó a cabo en dos bancos submareales de Ensis macha de Playa Villarino, en el Golfo San José, provincia de Chubut (Figura 1). El Golfo San José se extiende entre

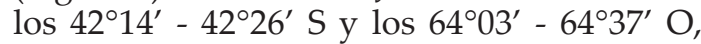
al norte de Península Valdés. Se encuentra separado del Golfo Nuevo por el istmo Ameghino y comunicado con el Golfo San Matías a través de una boca angosta de $9 \mathrm{~km}$ de ancho y $10 \mathrm{~m}$ de profundidad media. Es de forma elíptica, con su eje mayor en dirección este-oeste; su profundidad media es $38 \mathrm{~m}$. El régimen de mareas es semidiurno, con una amplitud media de 2.9 m (Rivas 1990). Los bancos seleccionados se denominaron banco control $\left(42^{\circ} 24^{\prime} 10.4^{\prime \prime} \mathrm{S}-64^{\circ} 17^{\prime} 25.1^{\prime \prime} \mathrm{O}\right)$ y banco impactado $\left(42^{\circ} 24^{\prime} 11.1^{\prime \prime} \mathrm{S}-64^{\circ} 17^{\prime} 28.8^{\prime \prime}\right.$ O), donde se realizó la actividad extractiva (Figura 1). La distancia entre bancos fue $85 \mathrm{~m}$. 


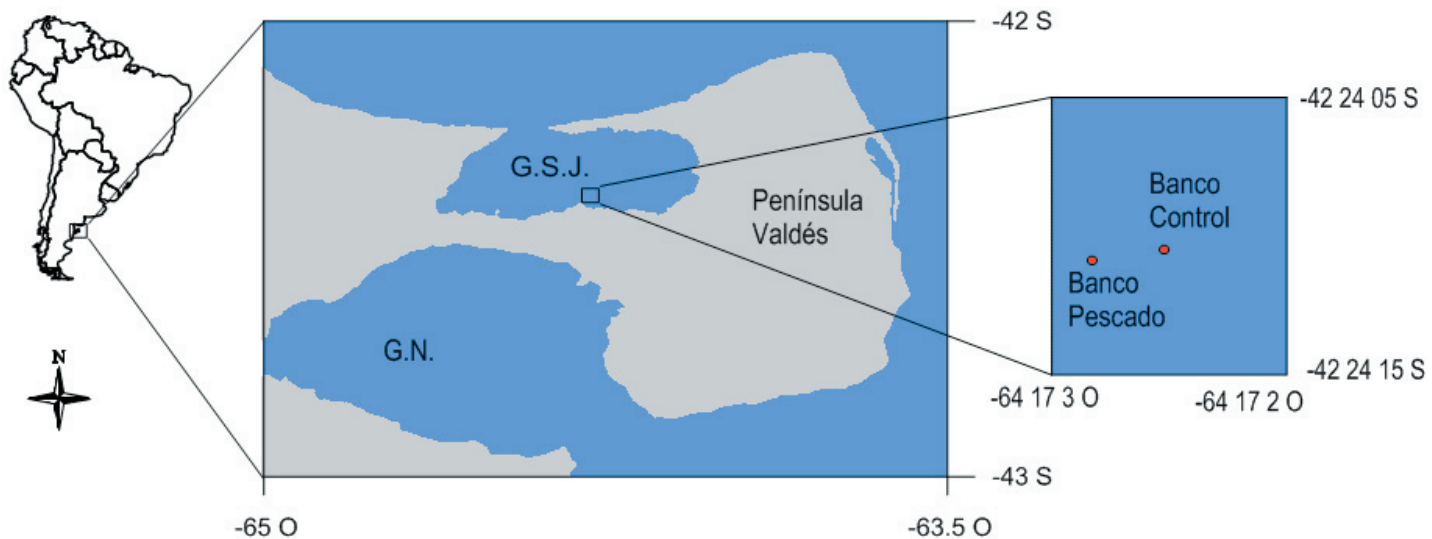

Figura 1. Mapa que muestra la localización geográfica de los bancos analizados. G.S.J.: Golfo San José. G.N.: Golfo Nuevo.

Figure 1. Map showing the location of the studied beds. G.S.J.: San José gulf. G.N.: Nuevo Gulf.

La elección de los bancos se realizó teniendo especial cuidado en que fueran similares en términos de características generales como la profundidad, el tipo de fondo, la composición del sedimento, la densidad de almejas y la intensidad de las corrientes, entre otras. Inicialmente, se planeaba repetir el evento de pesca en distintos bancos dentro del área de estudio $\mathrm{y}$, además, contar con un número equivalente de bancos control. Sin embargo, el diseño planteado previamente no se pudo completar por cuestiones logísticas (climáticas, sobre todo).

\section{Efecto de la pesca con hidrojet sobre las comunidades bentónicas}

Para evaluar el impacto de la pesca de almejas navajas mediante el uso de la técnica de hidrojet sobre las comunidades bentónicas se llevó a cabo una experiencia de pesca. En diciembre de 2011 se realizó un muestreo en los dos bancos seleccionados. Luego, se procedió a extraer navajas con hidrojet en uno de los bancos y al cabo de un año (enero de 2013) se evaluó la recuperación de la comunidad bentónica con un nuevo muestreo. El hidrojet es un arte de pesca que consiste en una bomba que se encuentra en la embarcación, que succiona agua de mar y la lanza a presión a través de una manguera manipulada por el buzo. El chorro de agua rompe la estructura del sedimento, lo satura con agua y así se pueden extraer las navajas. La cantidad de hoyos producidos por el hidrojet $\mathrm{y}$, por lo tanto, el alcance del disturbio, depende de la cantidad de almejas que se quieran extraer y de la densidad del banco. El área disturbada por la actividad extractiva durante la experiencia fue de $\sim 20$ $\mathrm{m}^{2}$. Para asegurar que el experimento de pesca realizado fuera representativo de los métodos que los pescadores aplican usualmente, se llevó a cabo con la colaboración y la supervisión de pescadores experimentados. Por otra parte, se realizaron visitas periódicas durante el año de recuperación para asegurar que ni en el banco control ni en el impactado se realizaran actividades extractivas, ni ocurriera ningún otro evento de perturbación. Las observaciones realizadas en estas visitas fueron de carácter cualitativo y no se incluyen en los análisis. En cada muestreo se colectaron diez muestras de fauna bentónica (macrobentos) por medio de buceo autónomo, para lo que se usaron cores de $10 \mathrm{~cm}$ de diámetro por 20 $\mathrm{cm}$ de profundidad ( $\mathrm{n}$ total $=40$ muestras). Para asegurar la independencia entre las muestras, tanto dentro del banco control como del impactado, se las colectó al azar dentro de cada banco, con una separación de al menos 1 $\mathrm{m}$. En el laboratorio, las muestras se tamizaron a través de una malla de $0.5 \mathrm{~mm}$. La fauna retenida se fijó en formol al $4 \%$ y se conservó en etanol al $70 \%$. Todos los organismos se identificaron taxonómicamente con un microscopio estereoscópico de disección (80x) y se estimó la abundancia de cada taxón por muestra. Los poliquetos fueron determinados hasta el nivel de familia, los moluscos en clase y los crustáceos hasta el nivel de orden.

Para determinar si existen diferencias significativas en la composición de las comunidades bentónicas entre el banco control y el impactado se utilizó un análisis de varianza con permutación (PERMANOVA), por medio de la extensión PERMANOVA del programa Primer v.6.1.7 (Anderson et al. 2008). El modelo de PERMANOVA incluyó 2 
factores fijos: banco (con 2 niveles: control y pescado) y tiempo (con 2 niveles: antes de la pesca y 1 año luego de la pesca) y utilizó 9999 permutaciones de los residuos bajo un modelo reducido. La homogeneidad de dispersión multivariada para los factores significativos del modelo de PERMANOVA se evaluó a través de la prueba PERMDISP. Luego, se realizaron comparaciones de a pares para el factor banco a fin de identificar en qué tiempos se produjeron las diferencias. Para explorar las similitudes y las diferencias entre los ensambles se usó un escalamiento multidimensional no métrico (nMDS) y se realizó un análisis de porcentaje de similitud (SIMPER) para determinar los taxa responsables de las diferencias entre los grupos. La abundancia de todos los taxa de invertebrados fue transformada con raíz cuarta con el fin de disminuir el peso de las especies más abundantes, y se utilizó una matriz de similitud de Bray-Curtis junto con una variable dummy.

\section{Resultados}

\section{Efecto de la pesca con hidrojet sobre las comunidades infaunales}

Considerando ambos tratamientos, se determinaron 24 taxa de invertebrados, representados por 15 familias de poliquetos, 2 clases de moluscos y 5 órdenes de crustáceos (Tabla 1). En el nMDS se observa que las muestras provenientes de los distintos bancos no se agruparon en grupos discretos para el tiempo inicial. Por el contrario, un año después de producido el disturbio se pueden distinguir 2 grupos bien definidos: uno que contiene a las muestras del banco control y otro con las muestras del banco afectado por la pesca con hidrojet (Figura 2). Las comunidades infaunales en el banco afectado por la pesca fueron significativamente diferentes a las del banco control (pseudo$\mathrm{F}_{\text {Banco }}=3.76$, perms $=9953, P($ perm $)<0.05$; pseudo- $\mathrm{F}_{\text {Tiempo }}=11.75$, perms $=9954, P($ perm $)$ $<0.05$; pseudo- $\mathrm{F}_{\text {BancoxTiempo }}=1.28$, perms $=9944$, $P($ perm $)>0.05)$. No existieron diferencias en la dispersión entre grupos en el factor banco $(\mathrm{F}=0.56, P>0.05)$. Si bien existieron diferencias en la dispersión entre grupos en el factor tiempo $(\mathrm{F}=50.52, P<0.05)$, el nMDS muestra una diferenciación de los centroides entre los distintos grupos. Las comparaciones de a pares mostraron que en el tiempo inicial (antes de la pesca) el banco control y el pescado presentaron una composición comunitaria similar $(\mathrm{t}=0.99, P($ perm $)>0.05$, perms $=6661)$, mientras que luego de 1 año de efectuarse la pesca por hidrojet, las comunidades de ambos

Tabla 1. Densidad media de los taxa de invertebrados (individuos $/ \mathrm{dm}^{3}$ ) para los dos bancos estudiados, antes del evento de pesca y luego de un año.

Table 1. Mean density of invertebrate taxa (individuals $/ \mathrm{dm}^{3}$ ) for the two studied beds, before and after one year of the fishing event.

\begin{tabular}{|c|c|c|c|c|c|}
\hline & & \multicolumn{2}{|c|}{ Banco control } & \multicolumn{2}{|c|}{ Banco pescado } \\
\hline & & Antes de la pesca & Al año & Antes de la pesca & $\mathrm{Al}$ año \\
\hline \multirow[t]{17}{*}{ Poliquetos } & Capitellidae & 6 & 4 & 3 & 1 \\
\hline & Cirratullidae & 1 & 2 & 0 & 2 \\
\hline & Glyceridae & 1 & 9 & 1 & 2 \\
\hline & Gonianidae & 0 & 1 & 0 & 0 \\
\hline & Hesionidae & 1 & 0 & 0 & 0 \\
\hline & Lumbrineridae & 6 & 11 & 6 & 5 \\
\hline & Maldanidae & 6 & 29 & 10 & 24 \\
\hline & Nephtydae & 1 & 3 & 2 & 0 \\
\hline & Nereidae & 0 & 0 & 0 & 1 \\
\hline & Onuphidae & 0 & 1 & 1 & 0 \\
\hline & Orbinidae & 1 & 1 & 1 & 0 \\
\hline & Paraonidae & 0 & 0 & 0 & 2 \\
\hline & Pilargiidae & 6 & 6 & 6 & 10 \\
\hline & Spionidae & 8 & 78 & 0 & 65 \\
\hline & Syllidae & 5 & 13 & 0 & 3 \\
\hline & Terebellidae & 0 & 4 & 0 & 0 \\
\hline & Phyllodocidae & 0 & 1 & 0 & 1 \\
\hline \multirow[t]{2}{*}{ Moluscos } & Bivalvia & 2 & 31 & 19 & 164 \\
\hline & Gasteropoda & 4 & 1 & 20 & 20 \\
\hline \multirow[t]{6}{*}{ Crustáceos } & Stomatopoda & 0 & 75 & 1 & 10 \\
\hline & Isopoda & 3 & 0 & 3 & 1 \\
\hline & Cumacea & 7 & 15 & 2 & 6 \\
\hline & Decapoda & 0 & 0 & 0 & 1 \\
\hline & Amphipoda & 1 & 82 & 4 & 23 \\
\hline & Tanaidacea & 1 & 9 & 0 & 0 \\
\hline
\end{tabular}


Tabla 2. Resultados del análisis SIMPER que muestran los taxa con las mayores contribuciones a la disimilitud en las comunidades del banco control y el banco afectado por la pesca luego de 1 año. Las listas fueron truncadas cuando el porcentaje acumulado alcanzó el $75 \%$.

Table 2. SIMPER routine results showing the taxa that made the greatest contributions to dissimilarity between control communities and the ones affected after 1 year of water-jet fishing. Lists were truncated when cumulative percentage reached $75 \%$.

\begin{tabular}{lccccc}
\hline & \multicolumn{2}{c}{ Abundancia promedio } & & & \\
Taxa & Control & Pesca & Disimilitud promedio & \% de contribución & \% acumulado \\
\hline Bivalvia & 4.8 & 25.8 & 20.81 & 29.07 & 29.07 \\
Spionidae & 12.2 & 10.2 & 12.75 & 17.82 & 46.89 \\
Amphipoda & 12.8 & 3.6 & 10.68 & 14.92 & 61.81 \\
Stomatopoda & 11.8 & 1.5 & 9.07 & 12.67 & 74.48 \\
Gasteropoda & 0.1 & 3.2 & 3.19 & 4.45 & 78.93 \\
\hline
\end{tabular}

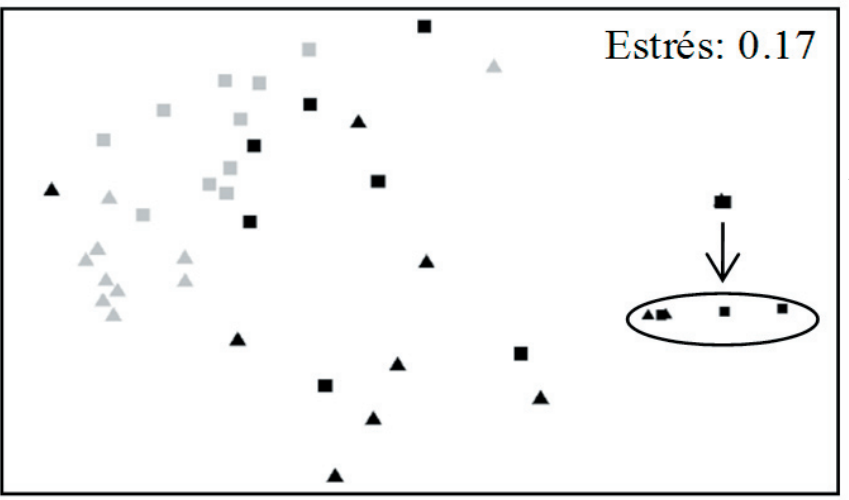

Figura 2. Análisis de ordenamiento bidimensional nMDS que compara los ensambles infaunales asociados a las muestras afectadas por la pesca (cuadrados) y control (triángulos) antes de la pesca por hidrojet (negro) y un año después de la pesca (gris).

Figure 2. Two-dimensional nMDS ordination comparing infaunal assemblages associated with fished-affected (squares) and control (triangles) samples before water-jet fishing (black) and one year after fishing (grey).

bancos difirieron significativamente $(\mathrm{t}=2.58$, $P($ perm $)<0.05$, perms $=9429)$. Luego de 1 año de pesca, los bancos presentaron una disimilitud promedio de $71.58 \%$ (análisis SIMPER). El banco control se caracterizó por la presencia de poliquetos de la familia Spionidae, anfípodos y estomatópodos, mientras que los moluscos bivalvos y gasterópodos fueron representativos en el banco afectado por la pesca (Tabla 2).

\section{Discusión}

El presente estudio aporta la primera evaluación experimental del efecto de la pesca con hidrojet de la la almeja navaja Ensis macha sobre las comunidades bentónicas en bancos submareales del sur de Sudamérica. El ensamble de invertebrados asociados a un banco almejas navajas afectado por la pesca con hidrojet fue diferente respecto a un banco cercano, utilizado como control. En diversos trabajos que estudian el impacto de la pesca con hidrojet se observó que los efectos inmediatos son más notorios que aquellos a largo plazo (Tuck et al. 2000; Hall et al. 1990) y atribuyen este hecho a la perturbación natural de los hábitats. Sin embargo, este no es el caso de los bancos de navajas estudiados, dado que las diferencias en la composición de las comunidades fueron persistentes en el tiempo, y se observaron diferencias entre las comunidades luego de un año de realizado el evento de pesca. Si bien es esperable una diferenciación natural entre los dos bancos analizados, el hecho de que las comunidades fueran similares en el tiempo inicial (antes del evento de pesca) permite suponer que la variación natural de estos hábitats es significativamente menor a la generada a raíz del disturbio producido por la pesca.

La pesca de la almeja navaja con hidrojet implica la remoción del sustrato mediante agua a presión. Los efectos de esta técnica afectan no sólo al sustrato, sino a las comunidades bentónicas asociadas a los bancos, ya sea al aumentar o al disminuir la abundancia de las especies presentes (Tuck et al. 2000). En concordancia con esto, la fauna bentónica del sitio pescado fue diferente al sitio control luego del evento de pesca; se registró una abundancia mayor de gasterópodos y bivalvos en el banco impactado (grupos que explican la mayor diferencia respecto al banco control). Así, los cambios producidos por la pesca con hidrojet en el ambiente estarían generando condiciones propicias que conducen a un aumento en la densidad de los mencionados taxa. En este sentido, los hábitos de vida de los 
taxa involucrados, por ejemplo, su modo de alimentación, se estarían viendo beneficiados por los cambios producidos con la remoción de las almejas mediante hidrojet. Por ejemplo, los bivalvos son organismos filtradores y podrían estar obteniendo una mayor cantidad de alimento gracias al movimiento de los sedimentos que produce este arte de pesca. Para conocer con precisión qué procesos estarían explicando el aumento de los grupos, deberían realizarse experimentos específicos en los que se puedan controlar las diferentes variables. En el caso de los gasterópodos, sería adecuado continuar con la determinación taxonómica para conocer la identidad de las diferentes especies presentes debido a la gran variabilidad de grupos tróficos dentro de la clase.

Las características físicas de los ambientes suelen influir en la distribución y abundancia de las especies, ya que modulan la disponibilidad de alimento, la movilidad de las especies y la disponibilidad de $\mathrm{O}_{2}$, entre otros. Así, factores como la profundidad, la salinidad, el tipo de sustrato, la composición granulométrica y la intensidad de las corrientes tienen gran incidencia sobre los distintos miembros de las comunidades bentónicas (Hall 1994; Lenihan and Micheli 2001; Díez et al. 2003). Si bien este trabajo no tuvo como objetivo investigar las características físico-ambientales de los sitios de estudio, al seleccionar los bancos se tuvo especial cuidado en que fueran similares en relación a las características ambientales principales y que la diferencia entre ambos se concentrara sólo en la simulación del evento de pesca. Dado que aquí no se presenta un análisis cualitativo de estas características físicas, los potenciales efectos entre estas propiedades y la comunidad bentónica asociada deberán ser abordados en estudios futuros. En este sentido, podría haber otros factores que estén afectando a las comunidades estudiadas, pero dada la cercanía entre los bancos y la similitud física entre los mismos, la influencia de estos factores (de existir) sería similar para ambos bancos.

Como se mencionó en Materiales y Métodos, por cuestiones logísticas no fue posible contar con diferentes bancos controles e impactados. Esta falta de repetición de bancos podría haber acotado el alcance de nuestros resultados, además de evitar proporcionar una idea de cómo es la distribución de las especies bentónicas en el área de estudio. Sin embargo, aun si los resultados obtenidos se suscriben a los dos bancos estudiados, son un punto de partida al momento de discutir la implementación a gran escala de la pesca mediante hidrojet y sus posibles impactos sobre las comunidades asociadas. Por otra parte, al conocer que los bivalvos en general y las almejas navajas en particular son capaces de modificar las condiciones hidrológicas y la dinámica y granulometría de los sedimentos (Sousa et al. 2009; Witbaard et al. 2016), habría sido interesante agregar al diseño experimental nuevos tratamientos para evaluar por separado el efecto de la remoción de navajas y el de los disturbios físicos (generados por el chorro de agua producido por el hidrojet). Sin embargo, dado el hábito de la navaja y su capacidad de enterrarse varios centímetros resulta imposible extraerlas sin disturbar el sustrato circundante. Para estudiar de manera individual el impacto del hidrojet se podría haber simulado el evento de pesca en un fondo donde no hubiera navajas, apartándonos del objetivo planteado. Así, las diferencias en la composición de especies halladas en nuestro trabajo son producto del efecto del hidrojet junto con el de la remoción de navajas.

Al momento de interpretar los resultados obtenidos en este trabajo se debe tener cierta precaución debido a la resolución taxonómica utilizada. En este sentido, el nivel taxonómico necesario y suficiente para cumplir con el objetivo de un estudio se denomina suficiencia taxonómica (del inglés "taxonomic sufficiency") (Ellis 1985); la decisión acerca de la resolución a la que los organismos deben ser identificados depende, esencialmente, del objetivo propuesto. La resolución usada aquí estuvo en concordancia con diferentes estudios en los cuales los efectos generados sobre las comunidades luego de un disturbio se detectan apropiadamente usando resoluciones taxonómicas superiores (familias $u$ órdenes) sin perder robustez en la evaluación del impacto (De Biasi et al. 2003; Bacci et al. 2009; Plicanti et al. 2016). Este enfoque es relevante a la hora de minimizar el tiempo de análisis (y los costos asociados) en función de obtener resultados, un punto clave en los estudios de evaluación de impacto ambiental. En nuestro trabajo, en caso de haber aumentado la resolución taxonómica empleada, cabría esperar encontrar diferencias mayores en la composición comunitaria de ambos bancos. Por ello, actualmente se estaría subestimando el efecto producido por la pesca, y no sobrestimándolo. De esta manera, los resultados obtenidos son conservadores al momento de sugerir recomendaciones de manejo. 
A partir de este trabajo se pudo observar que la remoción de navajas a partir de un solo evento de pesca con hidrojet produce cambios persistentes en el tiempo en las comunidades bentónicas asociadas a los bancos de almejas. Dado que el presente estudio no permite identificar los niveles sostenibles de perturbación, sería de gran utilidad llevar a cabo experiencias con eventos de pesca repetidos para generar un panorama más amplio del efecto de este tipo de disturbio sobre las comunidades. Los resultados hallados son claves para sugerir medidas de conservación y gestión a las autoridades locales a fin de evitar la degradación de los ambientes naturales y proteger la biodiversidad local.
Agradecimientos. Los autores agradecen al servicio de Náutica y Buceo del Centro Nacional Patagónico, en especial a Hormiga y Bebo. El estudio fue financiado por ANPCyTFONCyT (PICT 2010-0302 a SV) y la Secretaría de Ciencia, Tecnología e Innovación de la Provincia de Chubut (SCTeI N ${ }^{\circ}$ 10/11). El manuscrito fue mejorado en gran medida por los comentarios de dos revisores anónimos y del editor asociado Dr. Pedro Daleo. Agradecemos a las autoridades provinciales de Chubut por permitirnos trabajar dentro de Áreas Naturales Protegidas. Particularmente queremos destacar la colaboración y el asesoramiento de Marta Piñeiro y José Ascorti.

\section{ReFERENCIAS}

Almany, G. R. 2004. Does increased habitat complexity reduce predation and competition in coral reef fish assemblages? Oikos 106:275-284.

Anderson, M. J., R. N. Gorley, and K. R. Clarke. 2008. PERMANOVA+ for PRIMER: guide to software and statistical methods. PRIMER-E, Plymouth, United Kingdom.

Bacci, T., B. Trabucco, S. Marzialetti, V. Marusso, S. Lomiri, D. Vani, and C. Virno Lamberti. 2009. Taxonomic sufficiency in two case studies: where does it work better? Mar Ecol 30:13-19.

Bishop, M. J., C. H. Peterson, and H. C. Summerson. 2005. Effects of harvesting methods on sustainability of a bay scallop fishery: dredging uproots seagrass and displaces recruits Fishery Bull 103:712-719.

Ciocco, N. F. 1995. La marisquería mediante buceo en el Golfo San José (Chubut, Argentina). Informes Técnicos del PMIZCP, GEF-PNUD-FPN 21:1-39.

Ciocco, N. F. 2000. Almeja Panopea, un nuevo recurso pesquero para el mar argentino. Infopesca Int 6:36-39.

De Biasi, A. M., C. N. Bianchi, and C. Morri. 2003. Analysis of macrobenthic communities at different taxonomic levels: an example from an estuarine environment in the Ligurian Sea (NW Mediterranean). Est Coast Shelf Sci 58:99-106.

Díez, I., A. Santolaria, and J. M. Gorostiaga. 2003. The relationship of environmental factors to the structure and distribution of subtidal seaweed vegetation of the western Basque coast (N Spain). Est Coast Shelf Sci 56:10411054.

Ellis, D. 1985. Taxonomic sufficiency in pollution assessment. Mar Poll Bull 16:459.

Espinoza, R., J. Tarazona, and J. Laudien. 2010. Overfishing population characteristics of razor clam, Ensis macha, from Independencia Bay, Peru, in 2004 year. Revista Peruana de Biología 17:285-292.

Gaspar, M. B., R. Constantino, A. Guerra Díaz, and S. Carvalho. 2011. Environmental impacts of razor clam fisheries. Pp. 227-269 en Guerra Díaz, A., C. Lodeiros Seijo, M. B. Gaspar, and F. da Costa González (eds.). Razor clams: biology, aquaculture and fisheries. Xunta de Galicia.

Hall, S. J., D. J. Basford, and M. R. Robertson. 1990. The impact of hydraulic dredging for Razor clams Ensis sp. on an infaunal community. Netherland J Sea Res 27:119-125.

Hall, S. J. 1994. Physical disturbance and marine benthic communities: Life in unconsolidated sediments. Oceanography and Marine Biology: An Annual Review 32:179-239.

Lasta, M., N. F. Ciocco, C. Bremen, and A. Roux. 1998. Moluscos bivalvos y gasterópodos. Pp. 115-142 en Boschi, E. E. (ed.). El mar argentino y sus recursos Pesqueros, 2. INIDEP, Mar del Plata.

Lenihan, H. S., and F. Micheli 2001. Soft-sediment communities. Pp. 253-287 en Bertness, M. D., Gaines S. D. and Hay M. E. (eds.). Marine community ecology. Sunderland, M. A. Sinauer Associates, Inc.

Morsán, E., and N. F. Ciocco. 2011. Razor clam fishing in Patagonia, Argentina. Pp. 405-416 en Guerra Díaz, A., C. Lodeiros Seijo, M. B. Gaspar and F. da Costa González (eds.). Razor clams: biology, aquaculture and fisheries. Xunta de Galicia.

Rivas, A. L. 2009. Heat Balance and annual variation of mean temperature in the North-Patagonian gulfs. Oceanologica Acta 13:265-272.

Plicanti, A., R. Domínguez, S. F. Dubois, and I. Bertocci. 2016. Human impacts on biogenic habitats: Effects of experimental trampling on Sabellaria alveolata (Linnaeus, 1767) reefs. J Exp Mar Biol Ecol 478:34-44.

Robinson, R. F., and C. A. Richardson. 1998. The direct and indirect effects of suction dredging on a razor clam (Ensis arcuatus) population. ICES J Mar Sci 55:970-977.

Robledo, J., and S. Van der Molen. 2012. Fauna asociada a los bancos de almeja navaja en playa Villarino, Golfo San José (Chubut). VIII Jornadas Nacionales de Ciencias el Mar, Comodoro Rivadavia, Argentina.

Sousa, R., Gutiérrez, J. L., and D. C. Aldridge. 2009. Non-indigenous invasive bivalves as ecosystem engineers. Biol 
Invasions 11:2367-2385.

Tuck, I. D., N. Bailey, M. Harding, G. Sangster, T. Howell, N. Graham, and N. Breen. 2000. The impact of water jet dredging for razor clams, Ensis spp., in a shallow sandy subtidal environment. J Sea Res 43:65-81.

Witbaard, R., Bergman, M. J., van Weerlee, E., and G. C. Duineveld. 2016. An estimation of the effects of Ensis directus on the transport and burial of silt in the near-shore Dutch coastal zone of the North Sea. J Sea Res 127:95-104. 